\title{
Combination therapy with candesartan and lisinopril was more effective than monotherapy in type 2 diabetes and hypertension
}

\author{
Mogensen CE, Neldam S, Tikkanen I, et al. for the CALM Study Group. Randomised controlled trial of dual blockade of \\ renin-angiotensin system in patients with hypertension, microalbuminuria, and non-insulin dependent diabetes: the \\ candesartan and lisinopril microalbuminuria (CALM) study. BMJ 2000 Dec 9;321:1440-4.
}

\author{
QUESTION: What are the effects of candesartan or lisinopril, or both, on blood \\ pressure and the urinary albumin excretion rate in patients with hypertension, \\ microalbuminuria, and type 2 diabetes mellitus?
}

\section{Design}

Randomised (unclear allocation concealment*), double blind (investigators and patients),* controlled trial with 24 weeks follow up.

\section{Setting}

37 centres in tertiary hospitals and primary care sites (12 in Australia, 9 in Denmark, 4 in Finland, and 12 in Israel).

\section{Patients}

199 patients between 30 and 75 years of age with type 2 diabetes, microalbuminuria (urinary albumin to creatinine ratio between 2.5 and $25 \mathrm{mg} / \mathrm{mmol}$ ), and diastolic blood pressure (BP) between 90 and $110 \mathrm{~mm} \mathrm{Hg}$ while receiving placebo. Exclusion criteria included body mass index $\geqslant 40 \mathrm{~kg} / \mathrm{m}^{2}$, systolic BP $>200 \mathrm{~mm} \mathrm{Hg}$, non-diabetic cause of secondary hypertension, cardiovascular event in the previous 6 months, increased serum creatinine and potassium concentrations, and haemoglobin $\mathrm{A}_{1 \mathrm{c}}$ concentrations > 10\%. 197 patients (99\%) (mean age $60 \mathrm{y}, 65 \%$ men) had complete follow up.

\section{Intervention}

After a 4 week placebo run in period, patients were allocated to 1 of 4 groups: candesartan for 24 weeks $(\mathrm{n}=66)$, lisinopril for 24 weeks $(\mathrm{n}=64)$, candesartan for 12 weeks with the addition of lisinopril for a subsequent 12 weeks $(\mathrm{n}=34)$, or lisinopril for 12 weeks with the addition of candesartan for a subsequent 12 weeks $(\mathrm{n}=35)$. Patients in the latter 2 groups receiving combination regimens were combined in the 24 week analysis. Doses used were candesartan, $16 \mathrm{mg}$ once daily, and lisinopril, $20 \mathrm{mg}$ once daily.

\section{Main outcome measures}

$\mathrm{BP}$, urinary albumin to creatinine ratio, haemoglobin $\mathrm{A}_{1 c}$ concentrations, and adverse effects.

\section{Main results}

Analysis was by intention to treat. All treatments were effective in reducing $\mathrm{BP}$ and the urinary albumin to creatinine ratios. At 24 weeks, the mean reduction from baseline in diastolic BP was greater with combination treatment $(16.3 \mathrm{~mm} \mathrm{Hg})$ than with candesartan alone $(10.4 \mathrm{~mm} \mathrm{Hg}, \mathrm{p}=0.003)$ or lisinopril alone $(10.7 \mathrm{~mm}$ $\mathrm{Hg}, \mathrm{p}=0.005)$. Similarly, the mean reduction from baseline in systolic BP was greater with combination treatment $(25.3 \mathrm{~mm} \mathrm{Hg})$ than with candesartan alone $(14.1 \mathrm{~mm} \mathrm{Hg}, \mathrm{p}=0.002)$ or lisinopril alone $(16.7 \mathrm{~mm}$ $\mathrm{Hg}, \mathrm{p}=0.02)$. Combination treatment was associated with a greater mean reduction from baseline in urinary albumin to creatinine ratio than candesartan alone $(50 \%$ ข $24 \%, \mathrm{p}=0.04)$ but not lisinopril alone $(50 \%$ v $39 \%$, $\mathrm{p}>0.20)$. Groups did not differ for changes in mean haemoglobin $\mathrm{A}_{1 \mathrm{c}}$ concentrations. In general, all treatments were well tolerated.

\section{Conclusions}

Candesartan and lisinopril were effective monotherapies for reducing blood pressure and microalbuminuria; however, their combined use was well tolerated and more effective for reducing blood pressure in patients with type 2 diabetes, hypertension, and microalbuminuria.

*See glossary.
Source of funding: AstraZeneca, Mölndal, Sweden.

For correspondence: Dr C E Mogensen,

Kommunehospitalet, University Hospital, DK-8000 Aarhus $C$, Denmark. Fax +45 89492010.

\section{COMMENTARY}

Type 2 diabetes, hypertension, microalbuminuria, and cardiovascular disease are components of the insulin resistance syndrome. In many studies of patients with diabetes and microalbuminuria, angiotensin converting enzyme (ACE) inhibitors have been more effective than other agents in the short term reduction of microalbuminuria. Angiotensin II receptor antagonists have many properties that are similar to ACE inhibitors. However, they do not inhibit the breakdown of bradykinin, which causes the cough that is the most common side effect of ACE inhibitor treatment.

This study by Mogensen et al compared the effects of an angiotensin II receptor antagonist, candesartan, and an ACE inhibitor, lisinopril, on BP and microalbuminuria in patients with type 2 diabetes. Both drugs decreased BP and microalbuminuria, and the effect was even greater when the 2 drugs were combined. What remains unclear is whether similar benefits would have been obtained by increasing the dose of each agent; was the benefit for microalbuminuria because the renin angiotensin system was blocked more completely with both agents or because $\mathrm{BP}$ was reduced more effectively; or similar benefits might have been obtained if other agents had been added in combination.

The Hypertension in Diabetes Study (HDS) ${ }^{1}$ and Hypertension Optimal Treatment (HOT) trial $^{2}$ showed that aggressive treatment of hypertension in people with type 2 diabetes was associated with a reduction in cardiovascular outcomes. The 2 studies also showed that multiple hypotensive agents of different classes were needed to reach the target BP. Subsequently, the Heart Outcomes Prevention Evaluation (HOPE) study ${ }^{3}$ showed that the ACE inhibitor, ramipril, had cardiovascular benefits beyond reduction in BP. As a result, ACE inhibitors have become the drug of choice for treating hypertension in people with type 2 diabetes. The current study suggests that angiotensin II receptor antagonists should be considered if target $\mathrm{BP}$ is not reached. Trials are ongoing to determine whether angiotensin II receptor antagonists have benefits similar to those seen in the HOPE study and other trials.

Miles Fisher, MD

Glasgow Royal Infirmary, Glasgow, UK

1 UK Prospective Diabetes Study Group. Tight blood pressure control and risk of macrovascular and microvascular complications in type 2 diabetes: UKPDS 38. BMJ 1998;317:703-13. Erratum 1999;318:29

2 Hansson L, Zanchetti A, Carruthers SG, et al. Effects of intensive blood-pressure lowering and low-dose aspirin in patients with hypertension: principal results of the Hypertension Optimal Treatment (HOT) randomized trial. HOT Study Group. Lancet 1998;351:175562.

3 Heart Outcomes Prevention Evaluation Study Investigators. Effects of ramipril on cardiovascular and microvascular outcomes in people with diabetes mellitus: results of the HOPE study and MICRO-HOPE substudy. Lancet 2000;355:253-9. 